\title{
Multiplicity and transverse energy of produced gluon in relativistic heavy ion collision
}

\author{
Bo-Wen Xiad* \\ Department of Physics, Columbia University, New York, NY, 10027, USA
}

(Dated: November 20, 2018)

\begin{abstract}
We present a simple gluon production picture which is based on the McLerran-Venugopalan model and gluon BFKL evolution in relativistic heavy ion collision. Results for the multiplicity and transverse energy distribution in both the central and forward rapidity regions for gluon production in early stages of heavy ion collisions at the LHC are given. Finally, we provide a general qualitative discussion of the consequences of the forward rapidity behavior of produced gluons.
\end{abstract}

*Electronic address: bowen@phys.columbia.edu 


\section{INTRODUCTION}

The description of the early stages of heavy ion collisions before hadronization is currently an interesting and evolving topic in heavy ion physics, and it is still not clear how good a description can be obtained from Quantum Chromodynamics(QCD). However, a better understanding of the properties of the initially produced gluons would shed some light on the early stages of the physics in heavy ion collisions.

The McLerran-Venugopalan model[2] describes nuclear collisions at the Relativistic Relativistic Heavy Ion Collider (RHIC) in terms of the gluons in semi-classical nonabelian Weizsäcker-Williams field generated by the valence quarks of relativistic nuclei. At RHIC energies, it appears to be a good approximation since the actual gluon occupation number(gluon phase space density) is large enough to be described by the classical non-abelian Weizsäcker-Williams field. Specifically, we write the occupation number as $f_{g}=\frac{(2 \pi)^{3}}{2\left(N_{c}^{2}-1\right)} \frac{d N_{g}}{d^{3} b d^{3} k}=\frac{(2 \pi)^{3}}{2\left(N_{c}^{2}-1\right)} \frac{d N g}{d y d^{2} b d^{2} k_{-}}$. At LHC energies, quantum evolution in the longitudinal momentum (BFKL evolution[1]) should be more important and increase the number of gluons by increasing the saturation momentum while the occupation number $f_{g}$ does not change much 3] (we can see this either from the McLerran-Venugopalan model or from the discussion which follows.)

The Balisky, Fadin, Kuraev and Lipatov (BFKL) 1] equation is an equation which describes QCD hard scattering processes with a single hard transverse momentum scale and sums all terms like $(\alpha \ln (1 / x))^{n}\left(\right.$ or $\left.(\alpha Y)^{n}\right)$. Explicit evaluation of this equation tells us that how nucleus evolves when it is boosted to a larger rapidity, and this gives the rapidity evolution of the nuclear gluon distribution. Moreover, it has been shown [16] that the McLerran-Venugopalan model and fixed coupling BFKL evolution in the vicinity of the saturation boundary predicts geometrical scaling as shown below in Eq. (8). We suppose that this geometrical scaling is the right description for the nuclear gluon distribution beyond the saturation momentum, that is when $k_{1}>Q_{s}$, at LHC energies.

Based on a parton saturation model [4, 5, 6] and the color glass condensate [2, 7, 8], as well as a parton-hadron duality hypothesis, Kharzeev etal [9] have successfully made consistent calculation of hadron multiplicity in heavy ion collision, by assuming the produced gluons in the initial state hadronize into a corresponding number of hadrons in the final state. It appears that saturation and the Color Glass Condensate provide a reasonable physical 
pictures for relativistic heavy ion collisions. Therefore, these tools have begun to be used to describe and understand the thermalization stages of the produced gluons right after their formation during the heavy ion collision. Thus, a description of the produced gluon multiplicity and transverse energy distribution throughout all rapidity regions seems to be more and more important, and this is part of the motivation of this work.

In this paper, we are interested in the initial production processes of gluons in a heavy ion collision, which come from the freeing of the virtual gluons in colliding nuclei. The essential feature of this approach is that we choose our reference frame so that the produced gluon on which we are focusing has the rapidity $y$. Thus each of these two colliding nuclei have BFKL evolution in this particular frame. Based on the BFKL evolved gluon distribution in the nuclei and the approximate criteria [4, 11] developed by Blaizot and Mueller, we calculate the distribution of freed gluons from four different possible regions which are shown in Eqs. (10), (11), (12) and (13). In the end, we sum these contribution and obtain the total multiplicity and transverse energy distribution in Eqs. (15) and (17) for all produced gluons. In Eq. (15), we find that the first and the third terms are similar to the results in ref. [9] while the second term is different since we use geometrical scaling in the region beyond the saturation momentum. We also calculate the average transverse momentum per gluon in forward rapidity region. The calculations show that the average transverse momentum increases a lot while the number of produced gluons decreases rapidly in the forward rapidity region which may lead to some interesting physics in that region. As we will discuss in detail later, we consider the consequence of this result for jet quenching, gluon thermalization and elliptic flow. One of the conclusions is that we might expect the jet quenching effect to be less significant in forward rapidity region.

In section 2, we give a brief review of the McLerran-Venugopalan model, and derive the gluon distribution function in nuclei by following the interpretation of this model provided in ref. [10].

In section 3, we boost both of these two colliding nuclei (the projectile and the target) away from central rapidity region, and consider BFKL evolution[1] for both nuclei.

In section 4 , based on the framework established in above sections and the approximate criteria [4, 11] for gluon freeing, we calculate the rapidity dependence of the multiplicity and of the transverse energy of freed gluons.

Finally, in section 5, some qualitative discussions regarding the consequences of the for- 
ward rapidity behavior of produced gluons as well as a brief summary are provided.

\section{MCLERRAN-VENUGOPALAN MODEL}

Mueller and Kovechegov have shown[10] that in the McLerran-Venugopalan model, the gluon distribution of the relativistic nucleus can be written as following:

$$
\frac{d x G\left(x, k_{\perp}^{2}\right)}{d^{2} b d^{2} k_{\perp}}=\frac{N_{c}^{2}-1}{4 \pi^{4} \alpha N_{c}} \int \frac{d^{2} x_{\perp}}{\underline{x_{\perp}}}\left(1-e^{-\underline{x}^{2} Q_{s}^{2} / 4}\right) e^{-i k_{\perp}} \cdot \underline{x_{\perp}},
$$

in which the $k_{\perp}$ is the transverse momentum of the gluon, and the $x=\frac{k^{+}}{P^{+}}$is the longitudinal momentum fraction of the gluon. The saturation momentum, $Q_{s}$, is given by

$$
Q_{s}^{2}=\frac{8 \pi^{2} \alpha N_{c}}{N_{c}^{2}-1} \sqrt{R^{2}-b^{2}} \rho x g\left(x, 4 / \underline{x}^{2}\right)
$$

in which the $x g\left(x, 4 / \underline{x}^{2}\right)$ is just the gluon distribution of a single nucleon inside this nucleus. In the region $k_{\perp}^{2} \ll Q_{s}^{2}$, one can neglect the $\underline{x}^{2}$ dependence of the $Q_{s}^{2}$ and evaluate the above gluon distribution integral

$$
\frac{d x G\left(x, k_{\perp}^{2}\right)}{d^{2} b d^{2} k_{\perp}}=\frac{N_{c}^{2}-1}{4 \pi^{3} \alpha N_{c}} \int_{1}^{\infty} \frac{d t}{t} e^{-t k_{\perp}^{2} / Q_{s}^{2}}=\frac{N_{c}^{2}-1}{4 \pi^{3} \alpha N_{c}} \ln \left(\frac{Q_{s}^{2}}{k_{\perp}^{2}}\right) .
$$

Moreover, for a large nucleus using BFKL evolution, it has been found 12, 13, 14] that:

$$
\frac{d x G\left(x, k_{\perp}^{2}\right)}{d^{2} b d^{2} k_{\perp}}=\frac{N_{c}^{2}-1}{4 \pi^{3} \alpha N_{c}} \frac{1-\lambda_{0}}{2 \chi\left(\lambda_{0}\right)} \ln \left(\frac{Q_{s}^{2}}{k_{\perp}^{2}}\right),
$$

where $\lambda_{0}$ is defined by $\chi^{\prime}\left(\lambda_{0}\right)=-\frac{\chi\left(\lambda_{0}\right)}{1-\lambda_{0}}$, and $\chi\left(\lambda_{0}\right)=\psi(1)-\frac{1}{2} \psi\left(\lambda_{0}\right)-\frac{1}{2} \psi\left(1-\lambda_{0}\right)$ with the $\psi=\frac{d \ln \Gamma(\lambda)}{d \lambda}$ defined as the logarithmic derivative of the gamma-function. It is likely that the factors $\frac{N_{c}^{2}-1}{4 \pi^{3} \alpha N_{c}}$ and $\ln \left(\frac{Q_{s}^{2}}{k_{\perp}^{2}}\right)$ in the gluon distribution formula can be taken as general results except for an overall factor which could be fixed by a comparison to RHIC data. The essential physical picture is that the gluon density per unit area and per unit of twodimension transverse momentum, that is per unit of transverse phase space, is limited by (or saturates at) the product of a constant and logarithmic factor $\ln \left(\frac{Q_{s}^{2}}{k_{\perp}^{2}}\right)$ with a upper momentum limit $Q_{s}^{2}$. Therefore, one can write the gluon phase space density as

$$
\frac{d x G\left(x, k_{\perp}^{2}\right)}{d^{2} b d^{2} k_{\perp}}=\frac{k}{c} \frac{N_{c}^{2}-1}{4 \pi^{3} \alpha N_{c}} \ln \left(\frac{Q_{s}^{2}}{k_{\perp}^{2}}\right)
$$

in which $k$ is a $O(1)$ constant, and $c=\frac{2 \chi\left(\lambda_{0}\right)}{1-\lambda_{0}}=4.88$. In practice, one can change above gluon distribution into a uniform distribution as $\frac{d x G\left(x, k_{\perp}^{2}\right)}{d^{2} b d^{2} k_{\perp}}=\frac{k}{c} \frac{N_{c}^{2}-1}{4 \pi^{3} \alpha N_{c}}$, since the logarithmic factor disappears after integration over $k_{\perp}$. 


\section{BFKL EVOLUTION AND GEOMETRICAL SCALING}

However, the simple McLerran-Venugopalan (MV) gluon distribution does not contain any evolution in Bjorken $x$ which is necessary to explore the transverse energy spectrum. In order to extend our discussion from the central rapidity region to the forward rapidity region, we need to consider BFKL evolution of the saturation momentum. The leading order BFKL evolution for the saturation momentum is

$$
Q_{s}^{2}(A, Y)=Q^{2}(A) \frac{\exp \left[\frac{2 \alpha N_{c}}{\pi} \frac{\chi\left(\lambda_{0}\right)}{1-\lambda_{0}} Y\right]}{(\alpha Y)^{\frac{3}{2\left(1-\lambda_{0}\right)}}} .
$$

Numerical calculation shows that $\lambda_{0}=0.372$ and $c=\frac{2 \chi\left(\lambda_{0}\right)}{1-\lambda_{0}}=4.88$. This leads to the saturation momentum $Q_{s}^{2}(Y)$ goes like $\exp \left[\lambda_{s} Y\right]$ with $\lambda_{s} \sim 1$ if taking $\alpha=1 / 3$. This value is much larger than the phenomenological approaches 17] which find that $\lambda_{s}$ is about 0.3. Fortunately, the next to leading order calculation [18] shows that $\lambda_{s}$ effectively behaves like a constant around 0.30 since the next to leading correction slows down the evolution. Therefore, we shall take

$$
Q_{s}(A, y)^{2}=Q(A)^{2} \exp [0.30 y]
$$

in our numerical calculations.

Nevertheless, the above simple MV gluon distributions Eqs. (31), (41) and (51) are only valid when $k_{\perp}<<Q_{s}$, and they do not tell us how it behaves when the gluon transverse momentum $k_{\perp}$ is larger than the saturation momentum. Therefore, we need to consider the small-x evolution [1, 16] of the saturation momentum $Q_{s}$ and the gluon distribution in the vicinity of the saturation boundary. This gives a geometrical scaling of the gluon distribution beyond the saturation momentum scale [15, 16]. It has been shown [16] that beyond the saturation momentum, when $k_{\perp}>Q_{s}$, the evolved gluon distribution behaves like

$$
\frac{d x G\left(x, k_{\perp}^{2}\right)}{d^{2} b d^{2} k_{\perp}}=k \frac{N_{c}^{2}-1}{4 \pi^{3} \alpha N_{c}} \frac{1}{c}\left(\frac{Q_{s}^{2}}{k_{\perp}^{2}}\right)^{1-\lambda_{0}} \ln \left(\frac{k_{\perp}^{2}}{Q_{s}^{2}}\right) .
$$

We have chosen the same overall constant to agree with Eq. (15).

\section{PRODUCED GLUON MULTIPLICITY AND TRANSVERSE ENERGY}

In this section we use the McLerran-Venugopalan model together with BFKL evolution to investigate behavior of produced gluons in all rapidity regions. Let us concentrate on 


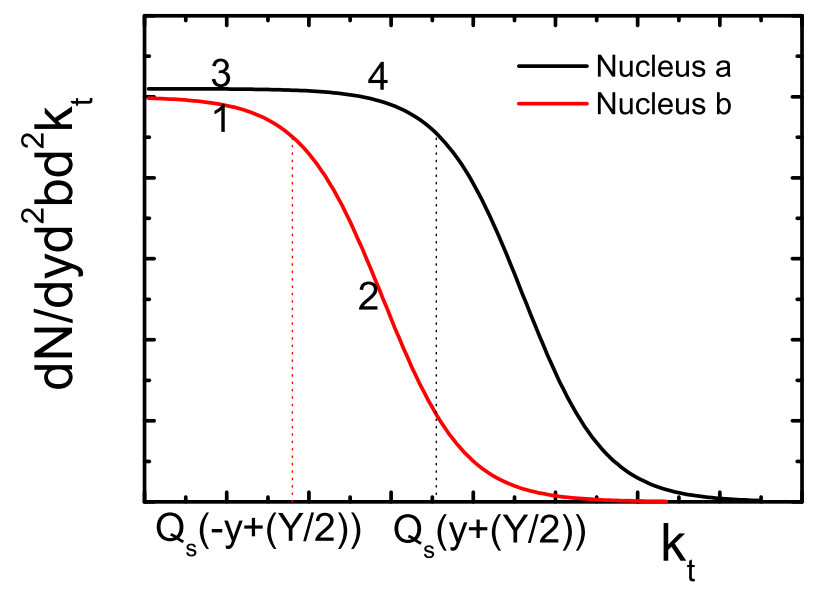

FIG. 1: Gluon distribution for two BFKL evolved nuclei, in which we denote the projectile(leftmoving) nucleus saturation momentum as $Q_{s}\left(y+\frac{Y}{2}\right)$ and target(right-moving) nucleus saturation momentum as $Q_{s}\left(-y+\frac{Y}{2}\right)$.

a simple case first, in which we consider the head on collision between the projectile(leftmoving) nucleus and target(right-moving) nucleus where both of the nuclei have the same number of nucleon; therefore the physics is symmetric under $y \Longleftrightarrow-y$. We also do not consider the impact parameter or centrality dependence for the moment. Thus for now we only consider the $y>0$ region.

The essential feature of this approach is that we choose a reference frame in which the produced gluons has zero rapidity. The two colliding nuclei then are assumed to have saturation momenta in the BFKL evolution region. Suppose the total rapidity difference between the target and projectile is $Y$, then the saturation momenta for the target and projectile nuclei are $Q_{s}\left(-y+\frac{Y}{2}\right)$ and $Q_{s}\left(y+\frac{Y}{2}\right)$ in this reference frame, respectively. Based on the BFKL evolved gluon distribution in the nuclei, we can determine the distribution of the freed gluons in our reference frame by using an approximate criteria 4, 11] developed by Blaizot and Mueller just as one usually does in the central rapidity region in the center of mass frame. The approximate criteria states that the gluons which receive enough transverse momentum to push them onto mass shell will be freed during the collision. As far as we know, the typical momentum of the gluons inside the nuclei are $Q_{s}\left(-y+\frac{Y}{2}\right)$ and $Q_{s}\left(y+\frac{Y}{2}\right)$ respectively. Correspondingly, the necessary typical transfer momenta in order to free gluons are $Q_{s}\left(-y+\frac{Y}{2}\right)$ and $Q_{s}\left(y+\frac{Y}{2}\right)$ respectively for these two nuclei as well. In this sense, we 


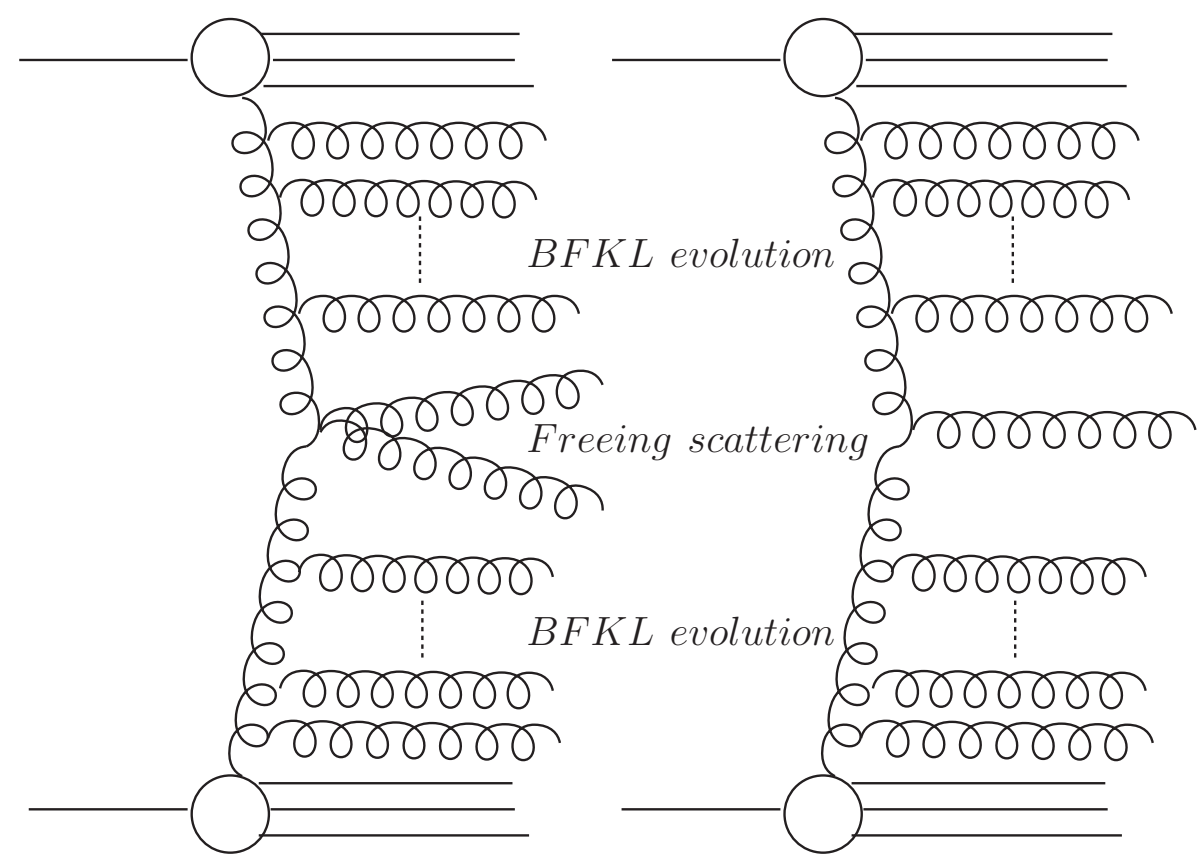

FIG. 2: Gluon BFKL evolution in the projectile and target nuclei as well as two possible freeing scattering process during the collisions.

can separate gluons inside the projectile and the target into four different according to these two saturation momenta as shown in Fig.(11).

1. Gluons inside the target with the typical saturation momentum $Q_{s}\left(-y+\frac{Y}{2}\right)$.

a. Gluons inside the target with a transverse momentum $k_{t}<Q_{s}\left(-y+\frac{Y}{2}\right)$ (Noting that $Q_{s}\left(-y+\frac{Y}{2}\right)<Q_{s}\left(y+\frac{Y}{2}\right)$ when $\left.y>0\right)$ are labeled as 1 in Fig.(11). They scatter with the gluons in the projectile nucleus which have a typical momentum $Q_{s}\left(y+\frac{Y}{2}\right)$ due to the gluon saturation and BFKL evolution which are illustrated in Fig.(2). During the scattering, the virtual gluons which are originally bounded inside the target nucleus are freed according to the approximate criteria that we have stated above. Therefore, those gluons in part 1 inside the target can be freed (or more properly, they are produced), and we calculate their total number per unit rapidity per unit area from the definition $\frac{d N_{1}}{d y d^{2} b}=\int_{0}^{Q_{s}\left(-y+\frac{Y}{2}\right)} d^{2} k_{\perp} \frac{d x G\left(x, k_{\perp}^{2}\right)}{d^{2} b d^{2} k_{\perp}}$ by integrating Eq. (4)

$$
\frac{d N_{1}}{d y d^{2} b}=\frac{k}{c} \frac{N_{c}^{2}-1}{4 \pi^{3} \alpha N_{c}} \pi\left(Q_{s}\left(-y+\frac{Y}{2}\right)\right)^{2} .
$$

Then, how are these produced gluons distributed in momentum space? Originally, we assume these gluons are almost uniformly distributed from QCD energy scale to the saturation momentum $Q_{s}\left(-y+\frac{Y}{2}\right)$ according to Eq. (4) and its simplified form where in Eq. (4) the 
logarithmic term is replaced by a constant. During the freeing process, these gluons exchange transverse momentum in the scattering with gluons from the projectile which have a typical momentum $Q_{s}\left(y+\frac{Y}{2}\right)$. This means that the momentum space distribution of these gluons has been enlarged from $Q_{s}\left(-y+\frac{Y}{2}\right)$ to $Q_{s}\left(y+\frac{Y}{2}\right)$. Therefore, we presume that these gluons would be uniformly distributed in the momentum space from very small transverse momentum (could be close to zero) to the typical momentum of the projectile nucleus $Q_{s}\left(y+\frac{Y}{2}\right)$ scale which is the typical momentum the freed gluons can acquire after the freeing. Then one finally obtain:

$$
\frac{d N_{1}}{d y d^{2} b d^{2} k_{\perp}}=\frac{k}{c} \frac{N_{c}^{2}-1}{4 \pi^{3} \alpha N_{c}}\left(\frac{Q_{s}\left(-y+\frac{Y}{2}\right)}{Q_{s}\left(y+\frac{Y}{2}\right)}\right)^{2},
$$

with $k_{t}<Q_{s}\left(y+\frac{Y}{2}\right)$. Note that the distribution of the freed gluons has been broadened from $k_{t}<Q_{s}\left(-y+\frac{Y}{2}\right)$ to $k_{t}<Q_{s}\left(y+\frac{Y}{2}\right)$ due to the scattering with the gluons from the projectile.

b. Gluons inside the target with a transverse momentum $k_{t}$ obeying $Q_{s}\left(-y+\frac{Y}{2}\right)<k_{t}<$ $Q_{s}\left(y+\frac{Y}{2}\right)$ are labeled as 2 in Fig.(11). They scatter with the gluons in the projectile nucleus which have a typical momentum $Q_{s}\left(y+\frac{Y}{2}\right)$. In this region, we believe that the distribution of the gluons inside the target obeys the geometrical scaling which we have shown in Eq. (8). Therefore, these gluons can be freed and we assume they are uniformly distributed from very small transverse momentum to the typical momentum scale of the projectile nucleus $Q_{s}\left(y+\frac{Y}{2}\right)$ scale after the freeing. The case is almost the same as the case a above except for the initial distribution of the gluons in the target. Then, we integrate over this region, and get the freed gluon distribution

$$
\frac{d N_{2}}{d y d^{2} b d^{2} k_{\perp}}=\frac{k}{c} \frac{N_{c}^{2}-1}{4 \pi^{3} \alpha N_{c}}\left\{\frac{1}{\lambda_{0}}\left(\left(\frac{Q_{s}\left(y+\frac{Y}{2}\right)}{Q_{s}\left(-y+\frac{Y}{2}\right)}\right)^{2 \lambda_{0}}-1\right)\right\}\left(\frac{Q_{s}\left(-y+\frac{Y}{2}\right)}{Q_{s}\left(y+\frac{Y}{2}\right)}\right)^{2} .
$$

with $k_{t}<Q_{s}\left(y+\frac{Y}{2}\right)$. Actually, we neglect the logarithmic term again in Eq. (8) before arriving at the result in \{\} in above equation while the exact result would be $\frac{1}{\lambda_{0}^{2}}\left[\left(\frac{Q_{s}\left(y+\frac{Y}{2}\right)}{Q_{s}\left(-y+\frac{Y}{2}\right)}\right)^{2 \lambda_{0}}\left(\ln \left(\frac{Q_{s}\left(y+\frac{Y}{2}\right)}{Q_{s}\left(-y+\frac{Y}{2}\right)}\right)^{2 \lambda_{0}}-1\right)+1\right]$. This simplification does not affect the physics much. In addition, we want to comment on the result that this is the largest contribution in the moderately large rapidity region due to the $\left(\frac{Q_{s}\left(y+\frac{Y}{2}\right)}{Q_{s}\left(-y+\frac{Y}{2}\right)}\right)^{\lambda_{0}}$ term which differs from the result in [9] although we both agree that this region gives largest contribution. This difference comes from the geometrical scaling, and we expect that heavy ion collisions at LHC might observe this scaling while at RHIC geometrical scaling may not yet 
set in since the saturation momentum may not be large enough.

c.Gluons inside the target with $k_{t}>Q_{s}\left(y+\frac{Y}{2}\right)$. In this region, the gluons are very few and the parton densities in the target are in the DGLAP evolution region. We believe this region does not contribute much.

2. Gluons inside the projectile with the typical saturation momentum $Q_{s}\left(y+\frac{Y}{2}\right)$.

a. Gluons inside the projectile with a transverse momentum $k_{t}<Q_{s}\left(-y+\frac{Y}{2}\right)$, labeled as 3 in Fig.(11), scatter with the gluons in the target nucleus which have a typical momentum $Q_{s}\left(-y+\frac{Y}{2}\right)$. Therefore, these gluons can be freed and we assume they uniformly distributed from very small transverse momentum to the typical momentum scale of the target nucleus $Q_{s}\left(-y+\frac{Y}{2}\right)$ after the freeing. Then one finds

$$
\frac{d N_{3}}{d y d^{2} b d^{2} k_{\perp}}=\frac{k}{c} \frac{N_{c}^{2}-1}{4 \pi^{3} \alpha N_{c}},
$$

with $k_{t}<Q_{s}\left(-y+\frac{Y}{2}\right)$. Note that the range of the momentum remains the same because the typical momentum that the freed gluons scatter with is $Q_{s}\left(-y+\frac{Y}{2}\right)$. If we integrate over two-dimension phase space in Eqs. (10) and (12), we get the same results with Kharzeev etal [9].

b.Gluons inside the projectile with a transverse momentum $Q_{s}\left(-y+\frac{Y}{2}\right)<k_{t}<Q_{s}(y+$ $\left.\frac{Y}{2}\right)$ are labeled as 4 in Fig.(11). They scatter with the gluons in the target nucleus whose distribution is given by geometrical scaling. The problem here is very similar to that of case 1.b. and we presume that the contribution for this part can be written as

$$
\frac{d N_{4}}{d y d^{2} b d^{2} k_{\perp}}=\delta \frac{d N_{2}}{d y d^{2} b d^{2} k_{\perp}}
$$

with $0<\delta<1$ and $k_{t}<Q_{s}\left(y+\frac{Y}{2}\right)$.

c. Gluons inside the projectile with $k_{t}>Q_{s}\left(y+\frac{Y}{2}\right)$. In this region the gluons are very few and the parton densities in the projectile are in the DGLAP evolution region. We believe that this region does not contribute much.

Now, when we generalize our discussion to the very forward rapidity region, the factor $x=$ $\frac{Q_{s}\left(A, \pm y+\frac{Y}{2}\right)}{\sqrt{s}} \exp [y]$ is no longer small. In this case we need to include a limiting fragmentation factor $(1-x)^{4}$ which comes from the phenomenological quark counting rules [19].

Then, we add all contribution together and integrate over the two-dimension phase space to get the total multiplicity with the fragmentation factor taken into account as a large $x$ 
correction

$$
\begin{aligned}
\frac{d N}{d y}= & \sum_{i=1}^{4} \int d^{2} b d^{2} k_{\perp} \frac{d N_{i}}{d y d^{2} b d^{2} k_{\perp}}(1-x)^{4} \\
= & \left(\frac{k}{c} \frac{N_{c}^{2}-1}{4 \pi^{3} \alpha N_{c}}\right)\left(\pi R^{2}\right)\left(\pi Q_{s}^{2}\left(A,-y+\frac{Y}{2}\right)\right) \\
& \times\left\{\left(1-\frac{Q_{s}\left(-y+\frac{Y}{2}\right)}{\sqrt{s}} e^{y}\right)^{4}\right. \\
& \left.+\left[\frac{1+\delta}{\lambda_{0}}\left(\left(\frac{Q_{s}\left(y+\frac{Y}{2}\right)}{Q_{s}\left(-y+\frac{Y}{2}\right)}\right)^{2 \lambda_{0}}-1\right)+1\right]\left(1-\frac{Q_{s}\left(y+\frac{Y}{2}\right)}{\sqrt{s}} e^{y}\right)^{4}\right\} .
\end{aligned}
$$

In the above equation we have made the approximation that $x=\frac{k_{\perp}}{\sqrt{s}} \exp [y]$ for the gluons with transverse momentum $k_{\perp}$ in the integral is so small that $(1-x)^{4}$ is always around 1 until $k_{\perp}$ approaches the upper limits $Q_{s}\left( \pm y+\frac{Y}{2}\right)$.

Beginning with the approximate criteria of freeing gluon, we finally arrive at almost the same result as Kharzeev etal [9] except for the second term in above equation since we are using the geometrical scaling gluon distribution when $k_{\perp}>Q_{s}$. We believe that the geometrical scaling distribution might be the right picture to describe gluon distribution beyond the saturation momentum at LHC energies [20].) Moreover, we also notice that the second term, which is the contribution from Eq. (111), is the largest term in forward rapidity region since it is enhanced by a factor of $\left(\frac{Q_{s}\left(y+\frac{Y}{2}\right)}{Q_{s}\left(-y+\frac{Y}{2}\right)}\right)^{2 \lambda_{0}}$. Then we can go one more step further, averaging over different transverse momenta to get the transverse energy distribution,

$$
\begin{aligned}
\frac{d E_{T}}{d y}= & \sum_{i=1}^{4} \int d^{2} b d^{2} k_{\perp} \frac{d N_{i}}{d y d^{2} b d^{2} k_{\perp}}(1-x)^{4} k_{\perp} \\
= & \left(\frac{k}{c} \frac{N_{c}^{2}-1}{4 \pi^{3} \alpha N_{c}}\right)\left(\pi R^{2}\right) \frac{2}{3}\left(\pi Q_{s}\left(A,-y+\frac{Y}{2}\right)^{2}\right) \\
& \times\left\{Q_{s}\left(-y+\frac{Y}{2}\right)\left(1-\frac{Q_{s}\left(-y+\frac{Y}{2}\right)}{\sqrt{s}} e^{y}\right)^{4}\right. \\
& \left.+Q_{s}\left(y+\frac{Y}{2}\right)\left[\frac{1+\delta}{\lambda_{0}}\left(\left(\frac{Q_{s}\left(y+\frac{Y}{2}\right)}{Q_{s}\left(-y+\frac{Y}{2}\right)}\right)^{2 \lambda_{0}}-1\right)+1\right]\left(1-\frac{Q_{s}\left(y+\frac{Y}{2}\right)}{\sqrt{s}} e^{y}\right)^{4}\right\}
\end{aligned}
$$

In addition, we should fix the parameters which appear in the multiplicity and transverse energy distribution before we plot $\frac{d N}{d y}$ and $\frac{d E_{T}}{d y}$ as a function of rapidity $y$ in fig. (3). We take the parameters to be $\alpha=1 / 3$ and $N_{c}=3$ according to convention, and we allow the parameter $\delta$ to vary from 0 to 1 in the plots. 

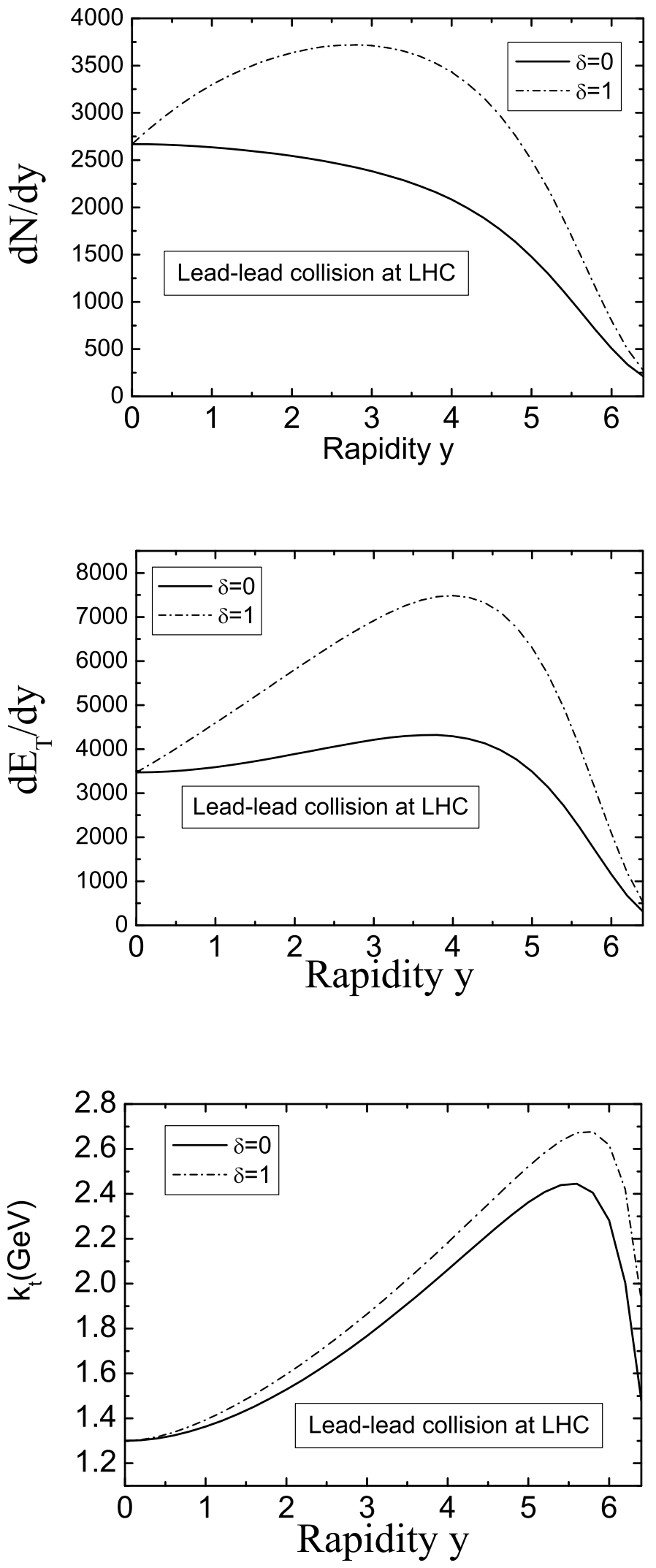

FIG. 3: Predictions of the multiplicity $\frac{d N}{d y}$, transverse energy distribution $\frac{d E_{T}}{d y}$ and average transverse momentum per gluon $\left\langle k_{\perp}(y)\right\rangle$ for the $\sqrt{s}=5500 \mathrm{GeV}$ lead-lead head-on collision at LHC when using $Q_{s}=1.95 \mathrm{GeV}$ and $\alpha=1 / 3$, respectively. 
Noting that $\exp \left[\frac{\lambda_{s} Y}{2}\right]=\left(\frac{\sqrt{s}}{M_{n u c}}\right)^{\lambda_{s}}$ where $M_{n u c}$ is the rest mass of one of the colliding nuclei, we can write the Eq.(17) as

$$
Q_{s}^{2}\left(A, y+\frac{Y}{2}\right)=Q_{s_{0}}^{2}(A)\left(\frac{\sqrt{s}}{\sqrt{s_{0}}}\right)^{\lambda_{s}} \exp \left[\lambda_{s} y\right] .
$$

Then, phenomenologically we take:

$$
\begin{aligned}
R & =r_{0} A^{\frac{1}{3}} \\
Q_{s}^{2}(A) & =(1.1 G e V)^{2}\left(\frac{A}{197}\right)^{\frac{1}{3}}\left(\frac{\sqrt{s}}{130 G e V}\right)^{\lambda_{s}}
\end{aligned}
$$

where $r_{0}=1.2 \mathrm{fm}$ is obtained from the empirical nuclear radius formula and $\left.Q_{s}\right|_{A=197}=$ $1.1 \mathrm{GeV}[3]$ is an estimation based on RHIC Au-Au collision at $\sqrt{s_{0}}=130 \mathrm{GeV}$ by using Eq. (21) and taking $\rho=\frac{A}{\frac{4}{3} \pi R^{3}}=\frac{1}{\frac{4}{3} \pi r_{0}^{3}}, x g\left(x, Q^{2}\right)=3.3$. Therefore, we predict that the saturation momentum at LHC is around $1.95 \mathrm{GeV}$ at $\sqrt{s}=5500 \mathrm{GeV}$ in the central rapidity region and $\pi R^{2}=158 \mathrm{fm}^{2}$ for lead nuclei from Eqs. (19) and (20). Noting that the geometrical scaling contribution in the multiplicity Eq. (15) vanishes in the $y=0$ central rapidity region means that $\left.\frac{d N}{d y}\right|_{y=0}$ should match RHIC data. Thus, we can fix the ratio of parameters $k$ and $c$ by using the multiplicity data in the central rapidity region from PHOBOS 21] which provides $\left.\frac{d N_{c h}}{d y}\right|_{y=0} ^{\sqrt{s}=130 \mathrm{GeV}}=547 \pm 55$ or $\left.\frac{d N}{d y}\right|_{\substack{\sqrt{s}=130 \mathrm{GeV} \\ y=0}}=821 \pm 83$ (Here we assume $\left.\frac{d N}{d y}\right|_{y=0}=\left.\frac{3}{2} \frac{d N_{c h}}{d y}\right|_{y=0}$ in which $\left.\frac{d N_{c h}}{d y}\right|_{y=0}$ is the multiplicity of charged particles, and we also neglect the difference between the rapidity $y$ and pseudorapidity $\eta$.). This helps us to fix $\frac{k}{c}=\frac{1}{2.34}$ which agrees with Mueller's conjecture[11](Mueller suggests that the total number of the produced gluons is proportional to the total number of the gluons in the initial nuclear wave-function and the proportionality coefficient $\epsilon$ is called gluon liberation coefficient.) and previous studies [22, 23, 24, 25] that the gluon liberation coefficient $\epsilon=\frac{2 k}{c}=\frac{2}{2.34}$ (this relation can be extracted from Eq. (15) via the definition $\left.\frac{d N}{d^{2} b d y}\right|_{y=0}=\epsilon \frac{N_{c}^{2}-1}{4 \pi^{3} \alpha N_{c}} \pi Q_{s}^{2}$ at central rapidity region.) is an order 1 coefficient.

Then with fixed coefficients and Eq. (15), we are able to predict that: $\frac{d N_{c h}}{d y} \mid \begin{aligned} & \sqrt{s}=200 G e V \\ & y=0\end{aligned}=$ 630 which agrees with PHOBOS data 26] $\left.\frac{d N_{c h}}{d y}\right|_{y=0} ^{\sqrt{s}=200 \mathrm{GeV}}=650 \pm 35($ syst $)$ and $\left.\frac{d N_{c h}}{d y}\right|_{y=0} ^{\sqrt{s}=5500 \mathrm{GeV}}=1780$ for the lead-lead collision in the LHC energies, which is close to Kharzeev's prediction $[9]\left(\frac{d N_{c h}}{d y} \mid \begin{array}{l}\sqrt{s}=5500 \mathrm{GeV} \\ y=0\end{array}=1750-2100\right)$.

Finally, for each rapidity, we calculate the average transverse momentum per gluon as

$$
\left\langle k_{\perp}(y)\right\rangle=\frac{\frac{d E_{T}}{d y}}{\frac{d N}{d y}}
$$


and plot $\left\langle k_{\perp}(y)\right\rangle$ also in Fig. (3).

We find that the average transverse momentum per parton in our calculation (as well as that in Kharzeev's paper if one generalize it and calculate the average transverse momentum) increases a lot as we move to the forward rapidity region. (When $y>6,\left\langle k_{\perp}(y)\right\rangle$ starts to decrease because of the nonlinear effect of the limiting fragmentation factor $(1-x)^{4}$. We do not expect our discussion to hold in this region since $x$ again is no longer small when $y>6$, even at LHC energies.)

However, what we find is very different from the forward rapidity result [27] from RHIC for the final state hadrons where the average transverse momentum per hadron is almost constant as rapidity increases. There might be two reasons to explain this discrepancy. First, at RHIC the typical Bjorken $x$ is less than $10^{-2}$, and becomes comparable to 1 in the forward rapidity region. Also, the saturation momentum for one of the colliding nuclei becomes very small. This makes the saturation picture not applicable to the RHIC data in that region. The second reason is that there exist several intermediate processes (e.g., thermalization[28] and hadronization) between the initial produced gluons and final measured particles. This may indicate that the direct use of the parton-hadron duality hypothesis might be questionable and dangerous in this case. In order to get the right picture from initially produced partons to the final state particles, we need to consider the intermediate processes like thermalization which distorts the transverse momentum distribution of produced partons.

Finally, we sketch how to deal with the case where the projectile and target have different number of nucleons(e.g. D-Au collision). As one can see, $\frac{d N}{d y}$ reaches its maximum when two saturation momentum are the same. This happens at $y=0$ in the $A_{1}=A_{2}$ case. While in the $\mathrm{D}-\mathrm{Au}$ collision, the critical rapidity can be obtained by setting

$$
\begin{aligned}
Q_{s}\left(A_{1},-y+\frac{Y}{2}\right)^{2} & =Q_{0}\left(A_{1}\right)^{2}\left(\frac{\sqrt{s_{1}}}{\sqrt{s_{0}}}\right)^{\lambda_{s}} \exp \left[-\lambda_{s} y\right] \\
& =Q_{s}\left(A_{2}, y+\frac{Y}{2}\right)^{2}=Q_{0}\left(A_{2}\right)^{2}\left(\frac{\sqrt{s_{2}}}{\sqrt{s_{0}}}\right)^{\lambda_{s}} \exp \left[\lambda_{s} y\right]
\end{aligned}
$$

and noting that the saturation momentum is proportional to $A^{\frac{1}{3}}$. 


\section{CONCLUSION}

In the following, we would like to qualitatively discuss a little bit about the consequences of the above results in forward rapidity region. As we have seen(presuming $\delta$ is small compared to 1), the number of the produced gluon and the transverse energy in per unit rapidity decrease considerably in forward rapidity region, while the transverse energy per gluon per unit rapidity increases a lot in forward rapidity region. This leads to some changes compared to the central rapidity region.

1. Jet quenching: Jet quenching is a very important signal for the existence of the quark gluon plasma (QGP) in relativistic heavy ion collisions. After the hard scattering between two partons, each of the two scattered partons evolves into a jet which consists of a leading particle together with a number of associate hadrons around the leading particle during hadronization. However, in the presence of dense strongly interacting matter (QGP) surrounding the hard scattering site, the scattered partons have induced gluon radiation due to multiple scattering with the plasma and this causes the jet to lose energy. This energy loss causes a suppression of the observed leading hadron spectrum. This is the jet quenching. However, since the number of the produced gluons is much less in the forward rapidity region than in the central rapidity region, the induced energy loss is smaller since bremsstrahlung gluon radiation and multiple scattering are expected to be less important. Therefore, we might expect that the jet quenching effect would be less significant in the forward rapidity region. However, in the very large rapidity region our discussion breaks down because of two reasons: first, the saturation is no longer applicable to the region where $x$ is large; the other reason is that most of the valence quarks are in this region after the collision. The valence quarks may give a significant contribution to partonic density and increase the induced strong interaction which then might result in more energy loss.

2. Gluon thermalization: Baier etal. 28] have discussed thermalization of the partons, after they are produced and before hadronization, in the bottom-up thermalization scenario. In the above picture, thermalization time can be expected to be considerably longer in the regions away from the central rapidity as compared to the thermalization at $y=0$.

3. The elliptic flow: The concept of the elliptic flow parameter $v_{2}[29,30,31,32]$ is introduced to reflect the anisotropy of the produced gluons that due to the spatial geometrical

azimuthal asymmetry in the non-central collision. With the similar picture and discussion 
for the jet quenching, we can see that the elliptic flow is usually generated at the partonic level prior to the hadronization. At RHIC, data exhibits a very strong elliptic flow and saturate the hydrodynamic limit. This suggests that very rapid and almost complete local kinetic equilibrium is reached. At LHC, elliptic flow is again expected to saturate the hydrodynamic limit. In the forward rapidity region at LHC energies due to the decrease of produced gluons, we may expect a smaller elliptic flow.

In the saturation picture, we apply the McLerran-Venugopalan model and the geometrical scaling to the computation of the multiplicity and transverse energy distribution for heavy-ion collisions at LHC. Results for the multiplicity and transverse energy distribution in the central rapidity region agree well with the RHIC experimental data and previous studies. Then we make predictions for the results of the produced gluons at the early stage of heavy ion collisions in both central and forward rapidity regions at the LHC energy scale. Noticing that hadronization and thermalization can distort both the multiplicity and transverse energy distribution, we need to consider these effects before we compare the results of the produced gluons with the data of the final state particles.

Acknowledgements I would like to thank my advisor Professor Alfred.H. Mueller for suggesting this work and numerous stimulating discussions. Without his patient guidance, this work would not be possible. I also want to thank Cyrille Marquet for reading this paper and providing helpful comments on this work.

[1] Y.Y. Balitsky, L.N. Lipatov, Sov. J. Nucl. Phys. 28 822(1978); E.A. Kuraev, L.N. Lipatov and V.S. Fadin, Sov. Phys. JETP. 45199 (1977); L.N. Lipatov, Sov. Phys. JETP. 63904 (1986).

[2] L. McLerran, R. Venugopalan, Phys. Rev. D 492233 (1994); L. McLerran, R. Venugopalan, Phys. Rev. D 493352 (1994); L. McLerran, R. Venugopalan, Phys. Rev. D 502225 (1994).

[3] A. H. Mueller, Nucl. Phys. A 715, 20 (2003) arXiv:hep-ph/0208278.

[4] J.-P. Blaizot, A.H. Mueller, Nucl. Phys. B 289847 (1987).

[5] L. V. Gribov, E. M. Levin and M. G. Ryskin, Phys. Rept. 100, 1 (1983).

[6] A.H. Mueller, J. Qiu, Nucl. Phys. B 268427 (1986); A.H. Mueller, Nucl. Phys. B 415373 (1994); A.H. Mueller, B. Patel, Nucl. Phys. B 425471 (1994); A.H. Mueller, Nucl. Phys. B 437107 (1995); Z. Chen, A.H. Mueller, Nucl. Phys. B 451579 (1995). 
[7] Y.V. Kovchegov, Phys. Rev. D 545463 (1996); Y.V. Kovchegov, Phys. Rev. D 555445 (1997); Y.V. Kovchegov, Phys. Rev. D 60034008 (1999); Y.V. Kovchegov, Phys. Rev. D 61 074018 (2000).

[8] J. Jalilian-Marian, A. Kovner, L. McLerran and H. Weigert, Phys. Rev. D 55, 5414 (1997) arXiv:hep-ph/9606337 ; J. Jalilian-Marian, A. Kovner, A. Leonidov, H. Weigert, Nucl. Phys. B 504415 (1997); J. Jalilian-Marian, A. Kovner, H. Weigert, Phys. Rev. D 59 014015 (1999); E. Iancu, L. McLerran, Phys. Lett B 510145 (2001); for a review see E. Iancu, A. Leonidov, L. McLerran, arXiv:hep-ph/0202270; and E. Iancu and R. Venugopalan, arXiv:hep-ph/0303204.

[9] D. Kharzeev, M. Nardi, Phys. Lett B 507121 (2001); D. Kharzeev, E. Levin, Phys. Lett B 52379 (2001); D. Kharzeev, E. Levin, M. Nardi, The onset of classical QCD dynamics in relativisitic heavy ion collisions, hep-ph/0111315 D. Kharzeev, E. Levin, L. Mclerran Phys. Lett B 56193 (2003);D. Kharzeev, E. Levin and M. Nardi, Nucl. Phys. A 747, 609 (2005) arXiv:hep-ph/0408050.

[10] Yu. V. Kovchegov and A.H. Mueller, Nucl. Phys. B 529451 (1998).

[11] A. H. Mueller, Nucl. Phys. B 572, 227 (2000) arXiv:hep-ph/9906322.

[12] A. H. Mueller, Nucl. Phys. B 558, 285 (1999) arXiv:hep-ph/9904404.

[13] A. H. Mueller, Nucl. Phys. B 643, 501 (2002) arXiv:hep-ph/0206216.

[14] E. Iancu and L. D. McLerran, Phys. Lett. B 510, 145 (2001) arXiv:hep-ph/0103032.

[15] A. H. Mueller and D. N. Triantafyllopoulos, Nucl. Phys. B 640, 331 (2002) arXiv:hep-ph/0205167.

[16] A.H. Mueller, Nucl. Phys. A 724223 (2003); E. Iancu, K. Itakura, L. McLerran, Nucl. Phys. A 708327 (2002).

[17] K. Golec-Biernat, M. Wüsthoff, Phys. Rev. D 59014017 (1999); K. Golec-Biernat, M. Wüsthoff, Phys. Rev. D 60114023 (1999); A. M. Stasto, K. Golec-Biernat and J. Kwiecinski, Phys. Rev. Lett. 86, 596 (2001) arXiv:hep-ph/0007192.

[18] D.N. Triantafyllopoulos, Nucl. Phys. B 640331 (2002).

[19] S.J. Brodsky, G.R. Farrar, Phys. Rev. Lett. 311153 (1973).

[20] Private communication with Professor A.H. Mueller.

[21] B. B. Back et al. [PHOBOS Collaboration], Phys. Rev. Lett. $\mathbf{8 7} 102303$ (2001) arXiv:nucl-ex/0106006. 
[22] A. Krasnitz and R. Venugopalan, Nucl. Phys. B 557, 237 (1999) arXiv:hep-ph/9809433; A. Krasnitz and R. Venugopalan, Phys. Rev. Lett. 84, 4309 (2000) arXiv:hep-ph/9909203.

[23] Y. V. Kovchegov, Nucl. Phys. A 692, 557 (2001) arXiv:hep-ph/0011252.

[24] A. Krasnitz, Y. Nara and R. Venugopalan, Nucl. Phys. A 717, 268 (2003) arXiv:hep-ph/0209269; A. Krasnitz, Y. Nara and R. Venugopalan, Phys. Rev. Lett. 87, 192302 (2001) arXiv:hep-ph/0108092.

[25] T. Lappi, Phys. Rev. C 67, 054903 (2003) arXiv:hep-ph/0303076.

[26] B. B. Back et al. [PHOBOS Collaboration], Phys. Rev. Lett. 88022302 (2002) arXiv:nucl-ex/0108009.

[27] BRAHMS collaboration(I.G. Bearden et al.), nucl-ex/0403050.

[28] R. Baier, A. H. Mueller, D. Schiff, D. T. Son, Phys. Lett. B 50251 (2001); R. Baier, A. H. Mueller, D. Schiff, D. T. Son, Phys. Lett. B 53946 (2002); R. Baier, A. H. Mueller, D. Schiff, D. T. Son, Nucl. Phys. A 698 217c (2002).

[29] J. Y. Ollitrault, Phys. Rev. D 46229 (1992).

[30] P. Huovinen, P. F. Kolb, U. W. Heinz, P. V. Ruuskanen and S. A. Voloshin, Phys. Lett. B 503 58 (2001) arXiv:hep-ph/0101136; P. F. Kolb, P. Huovinen, U. W. Heinz and H. Heiselberg, Phys. Lett. B 500232 (2001) arXiv:hep-ph/0012137.

[31] D. Teaney, J. Lauret and E. V. Shuryak, arXiv:nucl-th/0110037; D. Teaney, J. Lauret and E. V. Shuryak, Phys. Rev. Lett. 864783 (2001) arXiv:nucl-th/0011058.

[32] M. Gyulassy, I. Vitev and X.N. Wang Phys. Rev. Lett. 862537 (2001); X.N. Wang Phys. Rev. C 63054902 (2001). 